$\mathbb{T}$ periodica polytechnica

Civil Engineering

$51 / 1(2007) 3+8$

doi: 10.3311/pp.ci.2007-1.01

web: http://www.pp.bme.hu/ci

(c) Periodica Polytechnica 2007

RESEARCH ARTICLE

\section{Numerical and laboratory investigation of the hydrodynamic complexity of a river confluence}

\author{
Sándor Baranya / János Józsa
}

Received 2006-04-03

\begin{abstract}
The paper deals with the hydrodynamic investigation of the junction of two rivers in north-west Hungary in an urban area. The goal of the investigation was to study the flow conditions by means of a $3 D k-\varepsilon$ turbulence CFD model and hydraulic scale model. The project gave the opportunity to compare the outcome of the distorted scale model with the ones of the CFD model in field scale, in the distorted hydraulic model scale as well as in undistorted scale, converting all the results to real field scale by assuming Froude similarity. Overall flow patterns, the effect of distortion and the robustness of the $k-\varepsilon$ turbulence model in such complex confluence conditions were analysed. Satisfactory agreement was found except for the region straight downstream of the confluence where significant differences between laboratory and numerical results were seen due most probably to the complex swirling and shearing character of the flow. Large scale vortex formation interacting with anisotropic local turbulence would certainly need differential Reynolds stress turbulence closure.
\end{abstract}

\section{Keywords}

$3 D$ CFD modelling $\cdot k-\varepsilon$ turbulence closure $\cdot$ river confluence $\cdot$ Mosoni-Danube

\section{Sándor Baranya}

Department of Hydraulic and Water Resources Engineering, Budapest University of Technology and Economics, H-1521 Budapest, Hungary e-mail: baranya@ vit.bme.hu

\section{János Józsa}

Department of Hydraulic and Water Resources Engineering, Budapest University of Technology and Economics, H-1521 Budapest, Hungary

\section{Introduction}

River confluences usually present complex flow patterns and related bottom topography. Such features have been found also in a study of the junction of two rivers in Hungary in an urban area. One of them is a long, regulated secondary branch of the river Danube with a mean flow around $50 \mathrm{~m}^{3} / \mathrm{s}$ whereas the other is the river Raab with a similar mean discharge but a lot higher when flooding. The former approaches the confluence in a slight whereas the latter in a strong bend. These conditions give rise to two secondary flows rotating in the opposite sense. Furthermore, the difference in the flow velocities at the interface of the two rivers results in a strong, inherently unstable shear layer, evolving into large scale horizontal vortices. The interaction of these two mechanisms and 3D turbulence govern the water exchange, mixing and morphology at the confluence.

As the confluence region is going to be significantly reshaped in the framework of a comprehensive city plan, prevailing flows have been extensively investigated under the existing conditions both by means of a 3D $k-\varepsilon$ CFD model and hydraulic scale modelling. Calibrated and cross-validated models have served then to analyse and evaluate variants conceived in the urban planning project.

The project gave at the same time the challenging opportunity to compare the results of the distorted scale model with the ones of the CFD model in field scale, in the distorted hydraulic model's scale, and finally in undistorted scale. All the results were converted to field scale by the Froude similarity law. Comparisons were carried out in terms of velocity distributions. In this paper the effect of distortion and the robustness of the $k-\varepsilon$ turbulence model under such complex confluence conditions is analysed.

\section{Numerical Model}

The numerical model used in this study is the CFD code called SSIIM [1]. SSIIM is an abbreviation for Sediment Simulation In Intakes with Multiblock option. It solves the Reynoldsaveraged Navier-Stokes equations with the two-equation $k-\varepsilon$ turbulence closure (see e.g. Rodi, 1984 [4]) in three space dimensions to compute the water flow using the finite volume approach 
as discretization method (see e.g. Patankar, 1980 [2]).

The model uses the complete momentum equations in all the three directions thus resulting in a non-hydrostatic flow description. The governing equations are solved in a finite volume context by using the SIMPLE method (Patankar, 1980 [2]) on a three-dimensional, non-orthogonal curvilinear structured grid.

The Reynolds-averaged Navier-Stokes equations in an Einstein-type summation form are as follows:

$$
\frac{\partial U_{i}}{\partial t}+U_{j} \frac{\partial U_{i}}{\partial x_{j}}=\frac{1}{\rho} \frac{\partial}{\partial x_{j}}\left(-P \delta_{i j}-\rho \overline{u_{i} u_{j}}\right),
$$

where $U=$ time-averaged velocity; $u=$ velocity fluctuation; $P=$ pressure; $x_{j}=$ Cartesian space co-ordinates; $\delta_{i j}=$ Kronecker delta; $\rho=$ fluid density.

The eddy viscosity concept with $k-\varepsilon$ turbulence closure is used to model the Reynolds stress terms as follows:

$$
-\rho \overline{u_{i} u_{j}}=\rho v_{T}\left(\frac{\partial U_{i}}{\partial x_{j}}+\frac{\partial U_{j}}{\partial x_{i}}\right)-\frac{2}{3} \rho k \delta_{i j},
$$

where $v_{T}=$ eddy viscosity coefficient; $k=$ turbulent kinetic energy.

The $k$ turbulent kinetic energy is defined as

$$
k \equiv \frac{1}{2} \overline{u_{i} u_{i}} .
$$

Substituting the Reynolds stress terms into the Reynoldsaveraged Navier-Stokes equations one obtains

$\frac{\partial U_{i}}{\partial t}+U_{j} \frac{\partial U_{i}}{\partial x_{j}}=\frac{1}{\rho} \frac{\partial}{\partial x_{j}}\left[-\left(P+\frac{2}{3} k\right) \delta_{i j}+v_{T} \frac{\partial U_{i}}{\partial x_{j}}+v_{T} \frac{\partial U_{j}}{\partial x_{i}}\right]$.

In the model, in which $\varepsilon$ represents the rate of turbulent energy dissipation, a transport equation is solved both for $k$ and $\varepsilon$ as a result of which the eddy viscosity coefficient can be evaluated as

$$
v_{T}=c_{\mu} \frac{k^{2}}{\varepsilon} .
$$

The transport of $k$ is modelled by the following differential equation:

$$
\frac{\partial k}{\partial t}+U_{j} \frac{\partial k}{\partial x_{j}}=\frac{\partial}{\partial x_{j}}\left(\frac{v_{T}}{\sigma_{k}} \frac{\partial k}{\partial x_{j}}\right)+P_{k}-\varepsilon,
$$

where $P_{k}$ defines the production of $k$, and this term is expressed as

$$
P_{k}=v_{T} \frac{\partial U_{j}}{\partial x_{j}}\left(\frac{\partial U_{j}}{\partial x_{i}}+\frac{\partial U_{i}}{\partial x_{j}}\right) .
$$

The transport of $\varepsilon$ is modelled by the following differential equation:

$$
\frac{\partial \varepsilon}{\partial t}+U_{j} \frac{\partial \varepsilon}{\partial x_{j}}=\frac{\partial}{\partial x_{j}}\left(\frac{\nu_{T}}{\sigma_{\varepsilon}} \frac{\partial \varepsilon}{\partial x_{j}}\right)+C_{\varepsilon 1} \frac{\varepsilon}{k} P_{k}-C_{\varepsilon 2} \frac{\varepsilon^{2}}{k} .
$$

The constant values of the $k-\varepsilon$ turbulence model are (Rodi, 1984 [4])

$$
\begin{aligned}
& C_{\mu}=0.09 \\
& C_{\varepsilon 1}=1.44 \\
& C_{\varepsilon 2}=1.92 \\
& \sigma_{k}=1.0 \\
& \sigma_{\varepsilon}=1.3
\end{aligned}
$$

The above equations are valid inside the fluid flow in the free turbulence zone, but next to the boundaries the flow characteristics are calculated from the well known formula (Schlichting, $1979[5]):$

$$
\frac{U}{u_{*}}=\frac{1}{\kappa} \ln \left(\frac{30 y}{k_{s}}\right),
$$

where $U=$ boundary aligned velocity; $u_{*}=$ friction velocity; $\kappa=$ von Karman constant; $y=$ distance from the wall, and $k_{s}$ is the roughness height.

\section{Laboratory Model}

Hydraulic scale modelling was performed in the laboratory of the Environmental and Water Resources Research Institute (VITUKI). Due to space constraints and the fact that a rather large flow domain had to be investigated, a 1:75 vertical and $1: 150$ horizontal scale was adopted, thus resulting in a distortion rate of 2 (Fig. 11. As usual in such cases, the model followed Froude's law, the gravity and inertia being the dominating forces, as a result of which the scale factor for the horizontal length is $c_{H}=150$, for the vertical length is $c_{V}=75$, for the velocity is $c_{v e l}=c_{V}^{0.5}=8.66$, whereas scale factor for the discharge is $c_{Q}=c_{v e l} \cdot c_{H} \cdot c_{V}=97428$. Flow velocities were measured with micro-propeller current meter in a number of crosssections, at one or two depth points in the vertical due to effective shallowness. Surface velocity patterns were captured in the usual way by long-exposure photos of surface floats. Sediment transport and morphological changes were not considered.

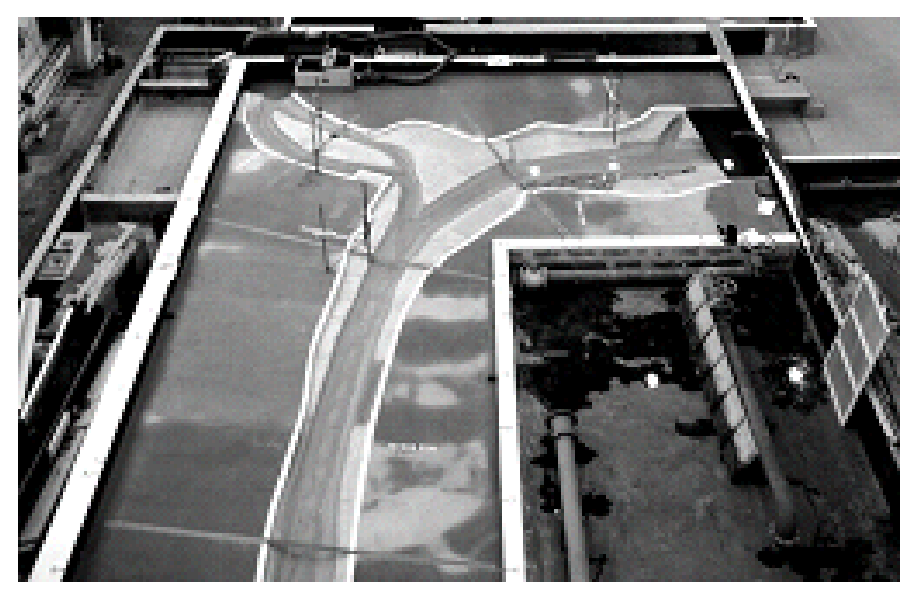

Fig. 1. The hydraulic scale model

\section{Study Area}

\subsection{Investigated River Reaches}

The investigated junction of the two rivers is situated in northwest Hungary in the city centre of Gyôr. One of them is MosoniDanube, a regulated secondary branch of river Danube with a mean flow of $50 \mathrm{~m}^{3} / \mathrm{s}$ and rather constant discharge all over the year due to the fact that it does not have any flood conveying role. It receives, however, significant backwater effect when Danube is flooding. The other is river Raab, which presents a natural, thus much more dynamic flow regime. Its mean flow 
is close to the one of the other river, but in case of flooding it can rise to several hundred $\mathrm{m}^{3} / \mathrm{s}$. The width of Mosoni-Danube in the study region is about $70 \mathrm{~m}$, while River Raab is somewhat narrower with $50 \mathrm{~m}$ on average. In mean flow conditions the depth of the rivers does not exceed $3 \mathrm{~m}$, except at some local scours.

The first step toward implementing the CFD model was to establish a reasonable digital terrain model. As an input for that, the digital elevation model (DEM) established on the basis of a recent bathymetry survey was used. Cross-sections for scale model building were taken from the DEM.

As can be seen in Fig. 2, the channel in Mosoni-Danube is characterized by near-shore berms due to considerable sediment deposition, while straight downstream of the tip of Radó-Island and in the vicinity of the junction scours have developed, the depth of which can reach even $8 \mathrm{~m}$.

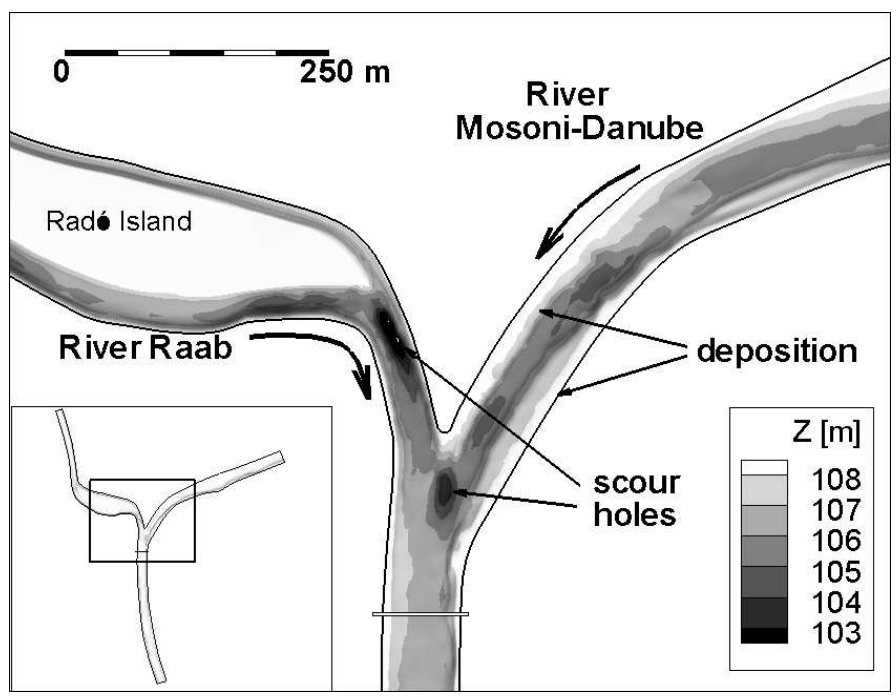

Fig. 2. The model sub-domain investigated more in details.

\subsection{Boundary Conditions}

Given that backwater profile in nature was measured at a discharge of $52 \mathrm{~m}^{3} / \mathrm{s}$ and $41 \mathrm{~m}^{3} / \mathrm{s}$ for Mosoni-Danube and River Raab, respectively, these discharge values were first used as upstream boundary conditions in model calibration. The water level corresponding to these discharge values was applied as downstream boundary condition, whereas the backwater curve inside the domain served to calibration.

Further investigated discharge combinations consisted of 40 $\mathrm{m}^{3} / \mathrm{s}$ for Mosoni-Danube, with $10 \mathrm{~m}^{3} / \mathrm{s}$ and $100 \mathrm{~m}^{3} / \mathrm{s}$ in River Raab, representing low and high flow conditions there, respectively.

A key model input is the parameterization of the roughness. The model offers several options to define this hydraulic resistance feature, out of which Strickler's smoothness coefficient was taken into account, resulting of course in an equivalent roughness height.

\subsection{Applied CFD Mesh}

The study reach of the river bed was mapped onto a single grid with 210 cells in stream-wise and 48 cells span-wise, fitted to the shoreline. In field scale it resulted in an average horizontal cell size of $6 \times 3 \mathrm{~m}$, while vertically 10 layers were used with reduced spacing toward the bottom to be able to capture strong gradients in the flow there.

For modelling flows in the designed bed geometry a grid with a bit higher resolution was applied with 280 cells in stream- and 60 cells in cross-wise direction. As the applied CFD model works on a structured grid the flow domain proper was finally defined by blocking out land-type cells (Fig. 3).

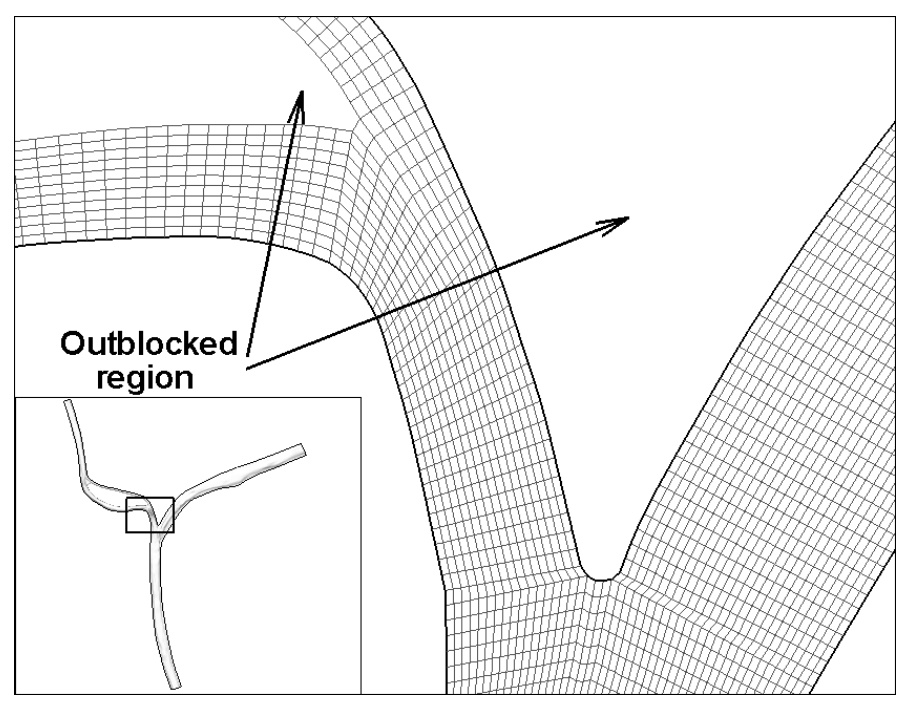

Fig. 3. Representative part of the applied mesh.

\section{Results and Discussion}

Both in laboratory and CFD modelling data of observed backwater curve and related 1D calculations served for overall smoothness calibration. The best fit in the CFD model was obtained with a smoothness of $40 \mathrm{~m}^{1 / 3} / \mathrm{s}$.

As mentioned in the introduction, the parallel laboratory and numerical investigations gave the challenging opportunity to compare and analyse the results of the following model variants:

1 Laboratory model with 1:75 vertical and 1:150 horizontal scale, respectively,

2 CFD model in the real field scale, in the hydraulic model's scale as well as in 1:75 undistorted scale.

As a common reference system, all results have been converted to real field scale according to Froude similarity law. As the free surface profiles showed acceptable fitting to each other, the main focus was on an inter-comparison of velocity distributions at selected characteristic cross-sections.

Characterizing turbulence conditions, while Reynolds number in field scale is in the order of $10^{6}$, at laboratory model scale $\operatorname{Re}_{l a b}$ was in the order of $1.5 \cdot 10^{3}$, certainly implying differences 
in primarily turbulence-driven processes and features as compared to the ones at the prototype scale. On the other hand, the presence of complicated swirling flows and spatial shear effects can reduce the chance of a $k-\varepsilon$ model to capture even the mean flow pattern due to the lack of direct modelling of anisotropy via Reynolds stresses in the applied turbulence closure.

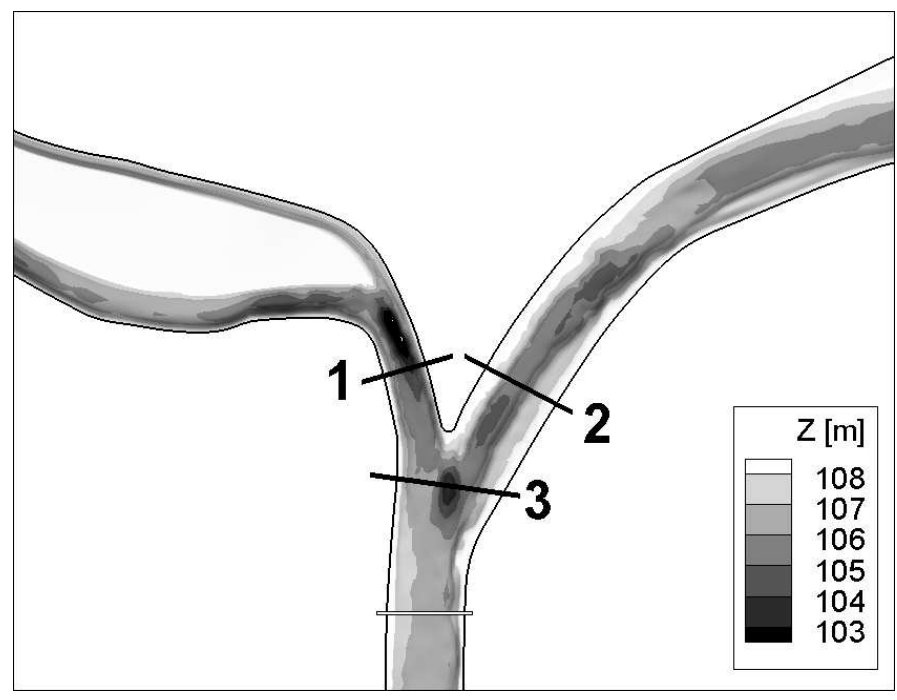

Fig. 4. Layout of cross-sections selected for illustrating modelled velocity distributions and secondary flow patterns.

Cross-sections selected for displaying calculated secondary currents and horizontal velocity distributions obtained by various approaches are indicated in Fig. 4. Velocity measurements taken by micro-propeller current meters at $1 \mathrm{~cm}$ below the surface in the scale model were used (equivalent to $0.75 \mathrm{~m}$ in field scale), and values interpolated to the same equivalent depth were extracted from the numerically modelled flow fields. The above mentioned velocity distributions corresponding to Section 2 and 3 in various discharge combinations and bathymetry variants are presented in Fig. 5. As it can be seen, the CFD calculations in general did not provide significant differences due to various space scaling in itself. Best fitting between scale model and CFD result was in principle expected when the numerical model ran for the laboratory scale. Agreement is indeed good enough at Section 2 upstream of the confluence, in rather smooth bathymetric and flow conditions. On the contrary, in Section 3 straight downstream of the junction significant differences between laboratory and numerical result can be seen, even for the case with identical space scaling.

In fact, the strong, complex swirling and shearing character of the flow pattern, interacting with anisotropic local turbulence, would certainly need even more sophisticated (e.g. differential Reynolds stress) closure to handle. The vicinity of such a river junction is just a reasonable test case to check numerical model capability. At the depth for which the comparisons were made the $k-\varepsilon$ type of closure provided differences up to $40 \%$ at places.

In order to have a better insight to the hydrodynamic complexity and its possible sources in the flow conditions, in the

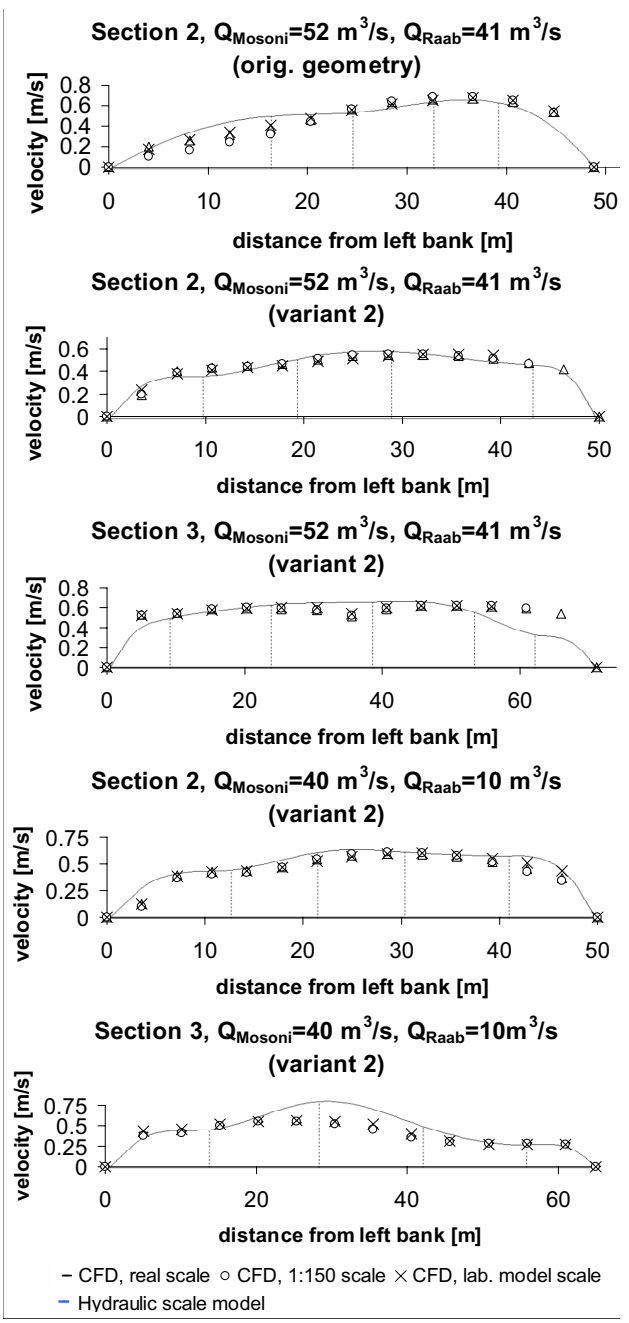

Fig. 5. Horizontal velocity distributions obtain in the hydraulic scale model as well as by various CFD approaches.

following further details of the flow features calculated in the study region are presented.

In Fig. 6 velocity vectors representing near-bottom (black) and near-surface (grey) flow magnitude as well as orientation in case of 52 and $41 \mathrm{~m}^{3} / \mathrm{s}$ discharge combination are displayed, as the branches approach the junction at various bending. As expected, significant secondary currents develop resulting in strong secondary current and overall stream-wise swirling of the flow, with the usual impact on the local cross-sectional shapes.

In River Raab the much larger branch seems to dominate the flow conditions downstream of the island tip, whereas in the confluence the two counter-rotating river streams join together by preserving the individual sense of rotation, however, creating a horizontally strongly shear interface layer (in fact, proved very unstable in the scale model, showing large scale KelvinHelmholtz instability).

Figs 7,8 show the flow velocity components in the plane of the selected cross-sections, both upstream and downstream of the junction. As the flow is just perpendicular to Section 1, a rather clear secondary flow pattern, along with the resulting shape can be seen there (with around $4 \mathrm{~m}$ as maximum depth). In Fig. 7, Section 2 shows cross-flows in the Mosoni-Danube. 
Fig. 6. Flow velocities near the bottom (black) and near the free surface (grey) in River Raab (left) and in the confluence zone (right).

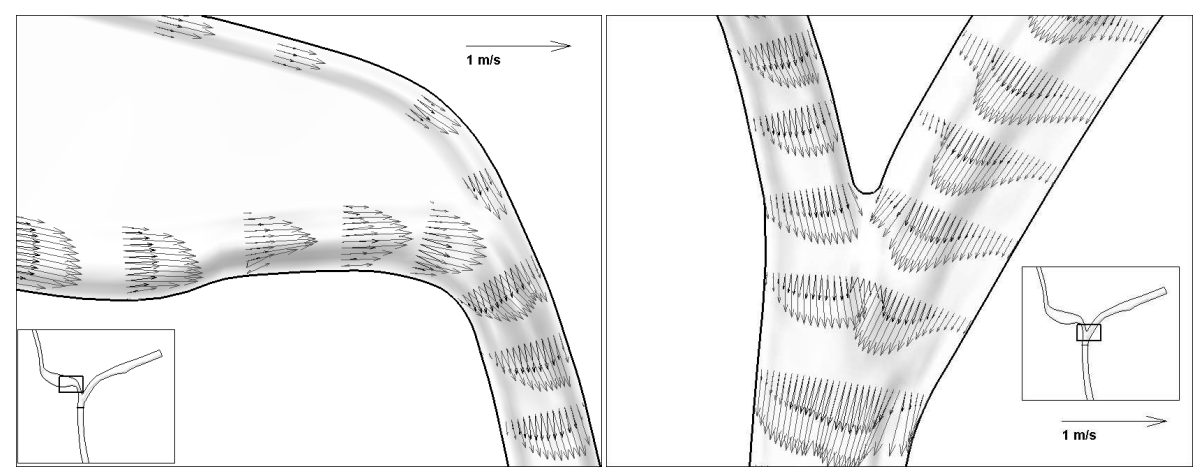

Fig. 7. Secondary flow pattern in River Raab in Section 1 (left) and in Mosoni-Danube in Section 2 (right).

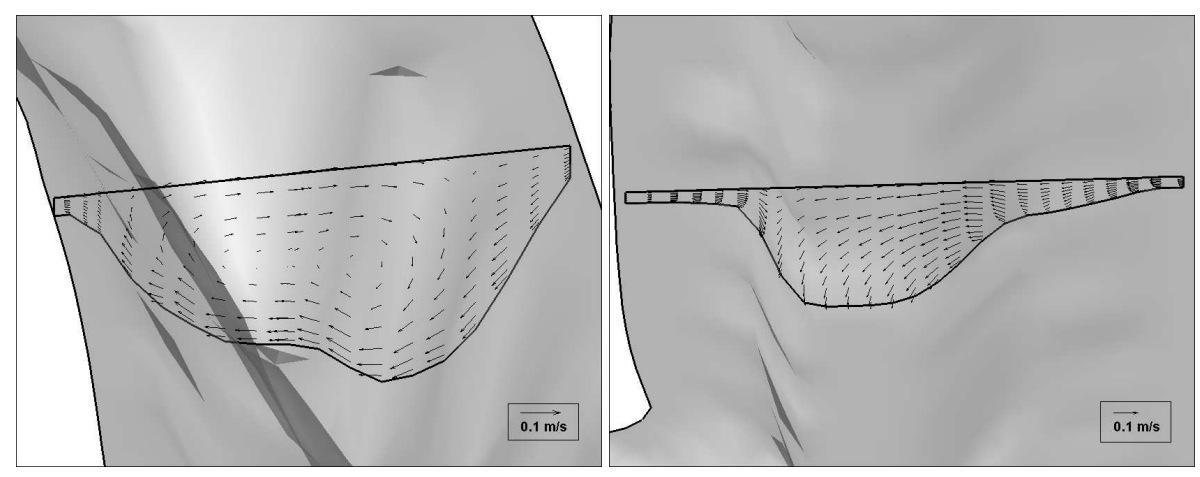

Flow in the near-shore zones is slow, resulting in considerable deposition to date.

In Fig. 8 one can then see the way the two swirling streams join by even mutually enhancing each other. The outcome of all that is strong downwelling in the sheared interface zone, what largely explains the development of a nearly $6 \mathrm{~m}$ deep local scour as a combined effect.

For the above discharge distribution, Fig. 9 provides a global view on the distribution of the flow velocity magnitudes, computed in planned bathymetry conditions (Variant 2, to reach sufficiently homogeneous cross-sectional velocity distribution), in real field scale as well as in distorted laboratory model scale.

Apart from the general character of the flow, one can also see the gradual, but at places rather abrupt restructuring of the spatial pattern. What can be seen, too, is that deviations between the results obtained in very different space extensions are not significant when converted using Froude similarity law. Remember, nevertheless, that it is a self-comparison, and the agreement in this location is thought to be further improved by means of more detailed turbulent stress calculation.

Of the indirectly derived flow features, vorticity fields were also analysed to quantify the complex shear in the interface zone of the two river streams. These near-surface vorticity fields are displayed in Fig. 10 for two bathymetry variants, showing less vortical, more uniform conditions in the planned conditions. Nevertheless, as it could be observed both in nature and in the lab, this layer inherently rolled up into large-scale, nearly horizontal eddies large enough even to be represented on the applied computational mesh. In fact, as the CFD model provides steady-state solutions, it is not entirely clear, to what extent of the kinetic energy of these 'Large Eddies' is represented by $k$ in the model, affecting in this way the entire local hydrodynamics.

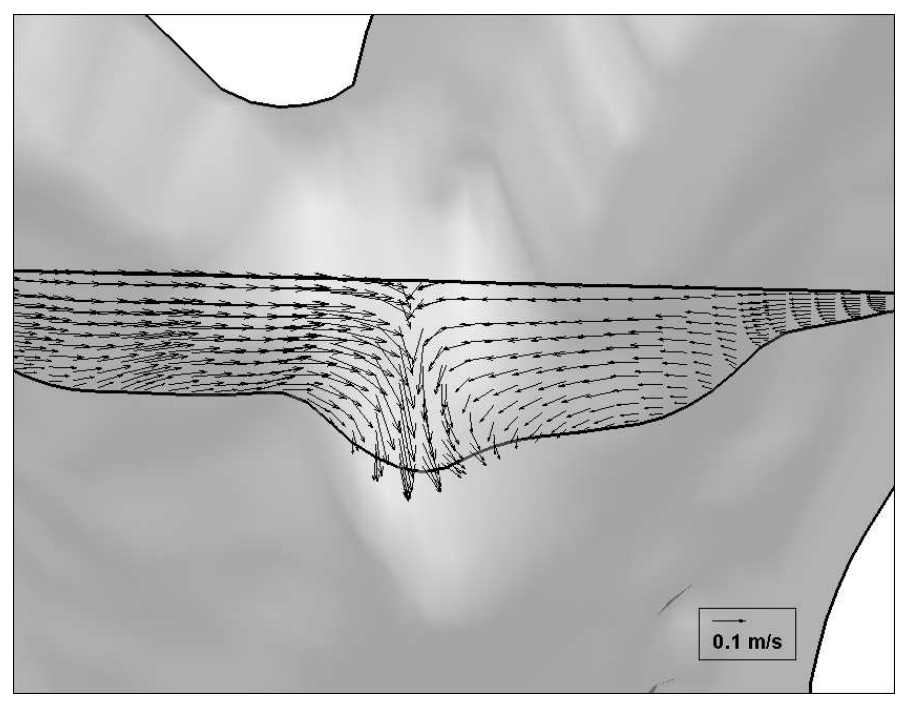

Fig. 8. Secondary flow pattern in the confluence zone (Section 3).

Introducing small perturbation into the unsteady version of the model thus inducing and maintaining such large scale coherent structures, or going for a LES approach might be the solution.

\section{Conclusions}

CFD and hydraulic scale models were simultaneously used for the hydrodynamic investigation of a typical junction of two moderate size rivers in Hungary. The laboratory model was set up with a distortion rate equal to 2 , nevertheless, still a bit shallow to obtain detailed quantitative measurements on the depthwise and spatial structure of the flow. Spatial distributions were investigated mainly by the CFD turbulence model, the validation of which was carried out by running the model also at the scale of the laboratory model. The CFD calculations in general provided slight differences due to various space scaling. Best fit between scale model and CFD result was expected to occur 
Fig. 9. Computed flow velocity magnitude distribution displayed at a number of cross-sections (left: Variant 2 - real field scale, right: Variant 2 - distorted laboratory scale).

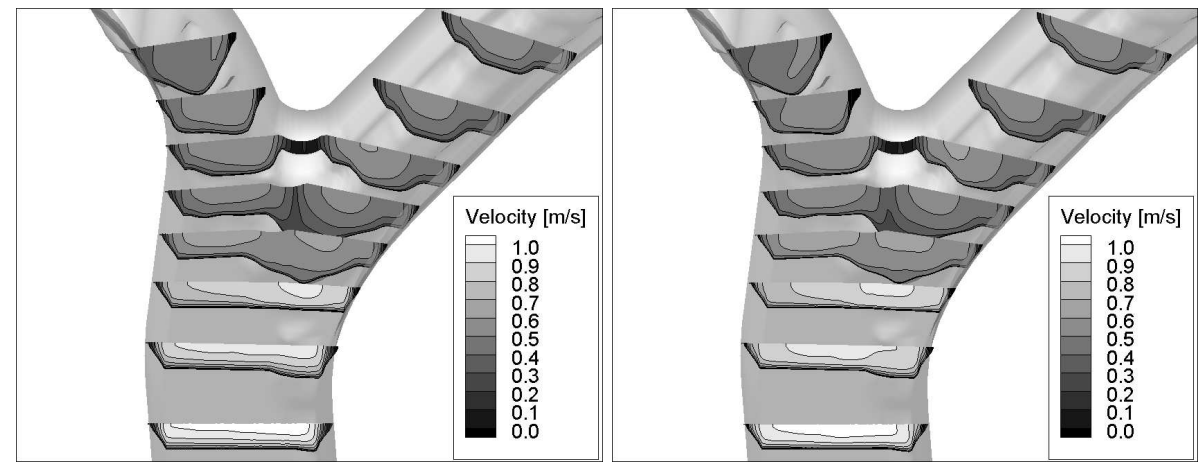

Fig. 10. Calculated near-surface vorticity magnitude distribution in the confluence zone (left: original bed geometry, right: variant 2).

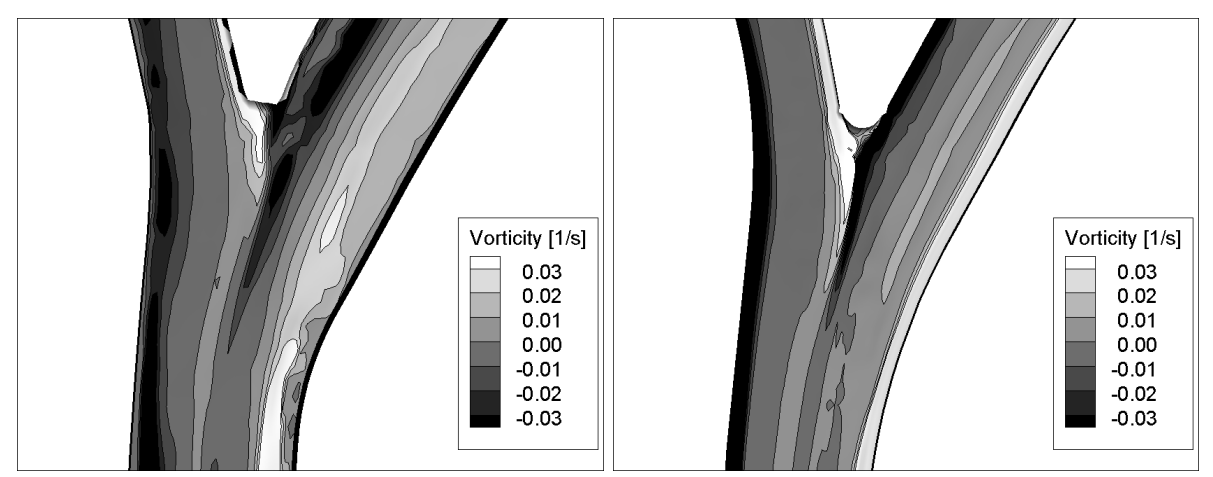

when the numerical model ran for the laboratory scale. Agreement was indeed good enough upstream of the confluence in rather smooth bathymetry and flow conditions. In turn, straight downstream of the junction significant differences between laboratory and numerical results were seen, even for the case with identical space scaling.

Strong, complex swirling and shearing character of the flow pattern, interacting with anisotropic local turbulence, would certainly need differential Reynolds stress closure there. Trying to initiate and maintain large-scale coherent structures in the existing model, or going to LES approach are also to be tried, along with detailed field measurements well-documented conditions, in order to move toward reliable morphological modelling as a next step.

\section{References}

1 Olsen NRB, A Three-dimensional Numerical Model for Simulation of Sediment Movements in Water Intakes with Multi-block Option: User's Manual, Department of Hydraulic and Environmental Engineering, The Norwegian University of Science and Technology, Trondheim, 2002.

2 Patankar SV, Numerical Heat Transfer and Fluid Flow, McGraw-Hill, New York, 1980.

3 van Rijn LC, Sediment transport, Part I: Bed load transport, Journal of Hydraulic Engineering, ASCE 110 (1984), no. 10, 1431-1456.

4 Rodi W, Turbulence Models and Their Application in Hydraulics - A stateof-the-art Review, International Association of Hydraulic Engineering and Research, Delft, 1984.

5 Schlichting H, Boundary Layer Theory, McGraw-Hill, New York, 1979. 